\title{
Comparative Modes of Failure: Trans-Sacral Rod Vs. Pedicle Screw for L5/ S1 Fusion - A Biomechanical Analysis
}

\section{Eric O. Klineberg1* ${ }^{1 *}$ Mark Kayanja ${ }^{2}$, Dhruv B. Pateder ${ }^{3}$ and Isador Lieberman ${ }^{4}$}

${ }^{1}$ Department of Orthopaedics, University of California at Davis, Sacramento, USA

${ }^{2}$ Orthopaedic Resident, The Cleveland Clinic Foundation, Cleveland, USA

${ }^{3}$ Orthopaedic \& Spinal Surgeon, Town Center Orthopaedic Associates, Reston, USA

${ }^{4}$ Orthopaedic \& Spinal Surgeon, Texas Back Institute, Plano, USA

\begin{abstract}
Introduction: The L5-S1 trans-sacral axial rod represents a less invasive approach to spinal fixation. No study to date has biomechanically tested this device to failure to compare it to the more common pedicle screw instrumentation in the human cadaveric spine.
\end{abstract}

Methods: Seven human lumbar spines (L4-pelvis) with an average $t$ score of -3.5 , were randomly divided into a pedicle screw construct [4] and axial trans-sacral rod [3]. The spines were then tested in a custom built platform to determine the effect of a pure flexion moment. Testing was performed with six cycles $(10 \mathrm{~N} / \mathrm{m})$, and final destructive testing (maximum $50 \mathrm{~N} / \mathrm{m}$ ).

Results: The pedicle screw construct has similar motion at the L5/S1 junction compared to the trans-sacral rod construct in cyclical testing. With destructive testing, no significant motion difference is observed at the L5/S1 segment, and no overt failure was noted. However, there was free motion and displacement at the L4/5 segment and in 3 specimens there was complete failure of the posterior ligamentous complex.

Conclusion: The trans-sacral axial rod was comparable to pedicle screws at the L5/S1 disc space in a pure flexion biomechanical model. No overt failure was observed in the destructive testing at the instrumented levels.

\section{Introduction}

While osteoporosis and spinal degeneration are independent processes, they frequently co-exist in older patients. Rigid fixation at an osteoporotic L5-S1 level is particularly difficult by virtue of the anatomy and biomechanics at this level. When the instrumentation fails at L5-S1, very few options exist for salvage or deformity correction. Rigid instrumentation may also have a greater propensity for failure in the osteoporotic spine due to the modulus mismatch between the bone and the implant. Many surgeons are now debating the role of less versus more instrumentation in the setting of osteoporosis [1].

With the recent advent of minimally invasive surgery, conventional thoughts and techniques regarding spinal instrumentation in osteoporotic bone are now being questioned. One of the main concepts of traditional spinal surgery has been to incorporate more fixation points in the spinal column to achieve a rigid construct. While this concept is valid, it is associated with extensive surgery, increased surgical morbidity, loss of spinal motion and a higher rate of complications [2].
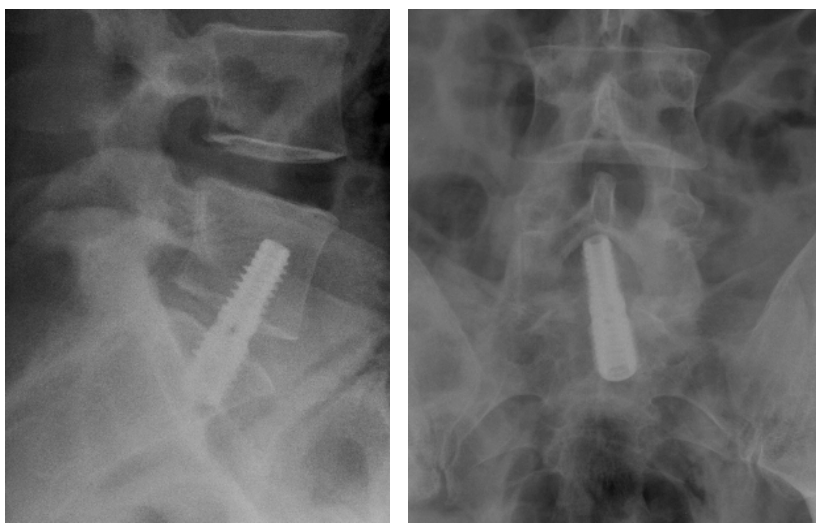

Figure 1: Post operative radiographs of Trans-Sacral Rod.
Perhaps the biggest problem with this fixation strategy is that when it fails, there are no other good revision options.

Novel instrumentation has been developed to improve the fusion rates at the L5/S1 motion segment. They combine the advantages of minimally invasive surgery with additional anterior support. In addition, these methods challenge the notion that more stability and more fixation equates to more predictable fusion and or better outcomes. The L5-S1 trans-sacral axial rod is such a device that can be placed at the L5-S1 junction using a less invasive percutaneous paracoccygeal trans-sacral approach (Figure 1). It is meant to provide effective fixation of the lumbosacral junction without disruption of the stabilizing posterior structures of the spine and the annulus of the intervertebral segment.

The purpose of this study was to biomechanically test and compare the degree of displacement at the L5/S1 level in motion segments instrumented with either the trans-sacral axial rod or posterior transpedicular screw fixation. We set out to test both cyclical flexion using physiologic loads, followed by a ramp to failure for both constructs. We hypothesized that the trans-sacral construct would have a biomechanical performance similar to the pedicle screw construct.

*Corresponding author: Eric O Klineberg, Department of Orthopaedics, UC Davis Health System, 4860 Y Street, Sacramento, CA 95817, USA, Tel: 916-734-2937; Fax: 916-734-7904; E-mail: eric.klineberg@ucdmc.ucdavis.edu

Received December 14, 2011; Accepted January 07, 2012; Published January 10, 2012

Citation: Klineberg EO, Kayanja M, Pateder DB, Lieberman I (2012) Comparative Modes of Failure: Trans-Sacral Rod Vs. Pedicle Screw for L5/S1 Fusion - A Biomechanical Analysis. J Spine 1:107. doi:10.4172/2165-7939.1000107

Copyright: ( 2012 Klineberg EO, et al. This is an open-access article distributed under the terms of the Creative Commons Attribution License, which permits unrestricted use, distribution, and reproduction in any medium, provided the original author and source are credited. 


\section{Materials and Methods}

Seven L4-S5 segments with attached pelvises were harvested from cadavers. The soft tissues were stripped off, sparing the annulus, ALL, PLL, supra and inter spinous ligaments, facet capsules and iliolumbar ligaments. The sacrum with attached L5 and L4 were separated from the remainder of the pelvis by division of the sacral ala $3 \mathrm{~mm}$ proximal to the SI joint. The segments were scanned using dual energy $\mathrm{x}$-ray absorptiometry (DEXA) to determine their bone mineral density (BMD) in grams per square centimeter with a Hologic QDR 4500A scanner (Hologic Inc., Waltham, MA). The specimens were then randomly divided into a group of four specimens instrumented with a pedicle screw construct (Abbot Spine, Austin, Tx.) and three instrumented with the AxiaLIF trans-sacral rod (TranS1 Inc., Wilmington NC). The pedicle screw L5/S1 construct was performed without interbody support. While anterior support is not necessary for L5/S1 fusion, in younger patients this is clinically more commonplace. However, in osteoporotic bone an inter-body graft may be more difficult to place safely without disruption of the endplates. Additionally, the trans-sacral rod was placed without posterior support i.e. pedicle screws or facet screws. We chose these models to represent the most disadvantageous biomechanical models especially for osteoporotic bone. All implants were inserted using fluoroscopy to ensure ideal placement. L4 and the sacrum were then embedded into polymethyl methacrylate (Fastray,
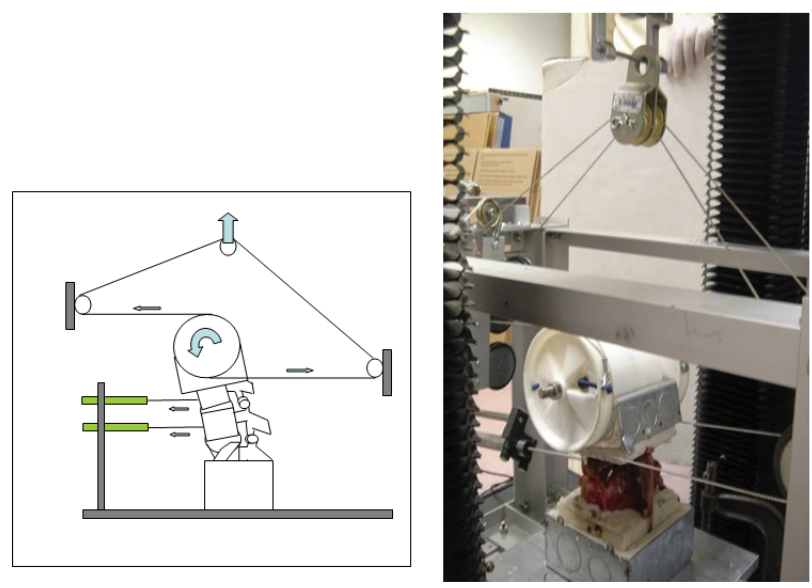

Figure 2: The custom testing platform.

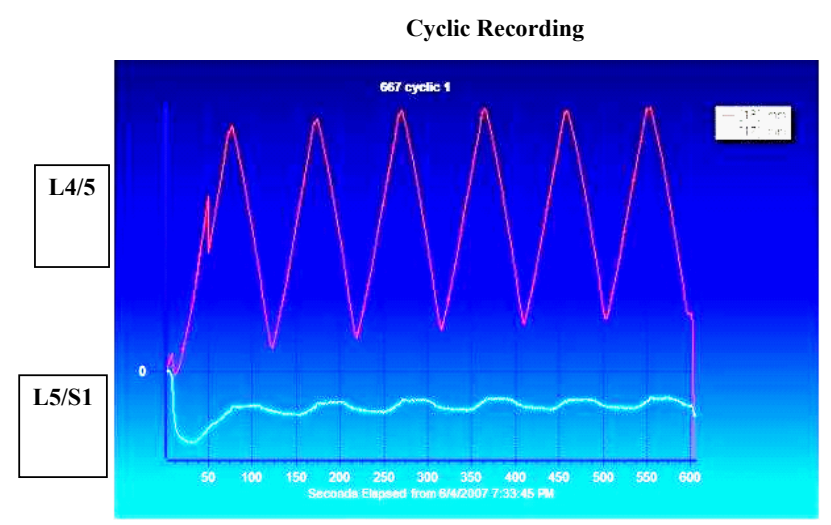

Figure 3: Representative cyclic data recorded from one of the pedicle screw constructs with 6 cycles at $10 \mathrm{Nm}$ over time.

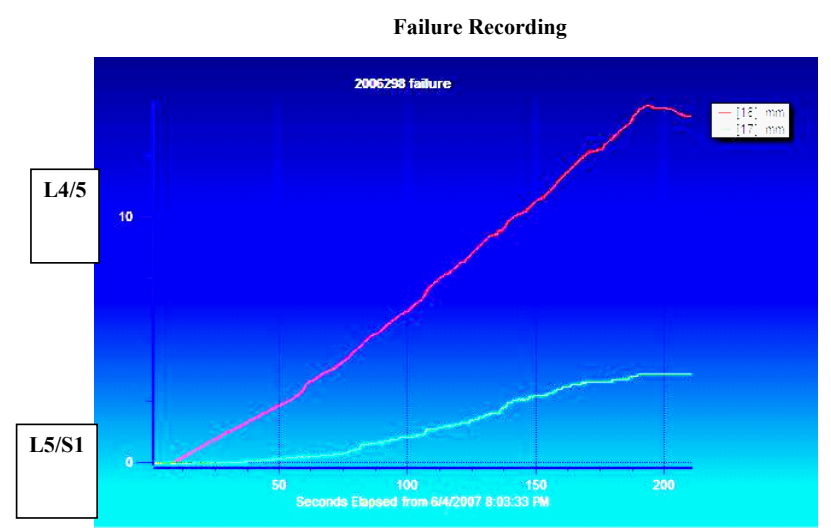

Figure 4: Representative pedicle screw construct tested to failure at maximum of $50 \mathrm{Nm}$

\begin{tabular}{|l|l|l|l|l|}
\hline & Group & Mean & Std. Deviation & N \\
\hline L4 / L5 & AxiaLIF & 2.179 & .5542 & 3 \\
\hline & Pedicle & 2.794 & .9077 & 4 \\
\hline & Total & 2.531 & .7891 & 7 \\
\hline L5 / S1 & AxiaLIF & .263 & .1494 & 3 \\
\hline & Pedicle & .086 & .0940 & 4 \\
\hline & Total & .162 & .1442 & 7 \\
\hline
\end{tabular}

Table 1: Cyclic statistics.

\begin{tabular}{|l|l|l|l|l|}
\hline & Group & Mean & Std. Deviation & N \\
\hline L4 / L5 & AxiaLIF & 16.660 & 8.9331 & 3 \\
\hline & Pedicle & 17.889 & 8.3571 & 4 \\
\hline & Total & 17.362 & 7.8709 & 7 \\
\hline L5 / S1 & AxiaLIF & 3.736 & 3.3353 & 3 \\
\hline & Pedicle & 4.572 & 4.1444 & 4 \\
\hline & Total & 4.214 & 3.5349 & 7 \\
\hline
\end{tabular}

Table 2: Failure statistics.

Bosworth, Skokie, IL) using custom aluminum squares which were gripped in the custom built platform. Extreme care was taken when potting the implants to prevent any cement support of the implants. This was accomplished in two ways. In the trans-sacral rod construct the insertion point was protected from impregnation with cement after instrumentation. This insured that no cement was in contact with the implant. Similarly, when potting the pedicle screw construct, the screws were not supported by any of the potting cement or aluminum squares. The potted spines were then placed into the custom aluminum squares and secured so that the implants were free and the anterior aspect of $\mathrm{S} 1$ was also free.

Testing was performed on this platform and was controlled by the materials testing machine (RT 10 Alliance MTS Inc., Eden Prairie). The platform was designed to provide a pure flexion moment (Figure 2). Motion was recorded using two AC excited spring loaded linear variable differential transformer (LVDT- Omega Engineering Inc., Stamford CT) that were attached to the L4 and L5 vertebral bodies. The linear motion was recorded at the L4/5 and L5/S1 segments and plotted. The cyclic deviation was then recorded and analyzed. The specimens were first tested in 6 cycles of flexion of 6 cycles from 0-10 Nm (Figure $3)$. This was followed by destructive testing with a progressively incremental moment to a maximum of $50 \mathrm{Nm}$ (Figure 4). Data was recorded and analyzed on Strainsmart 4.01 (Micromeasurements Group, Raleigh NC). 


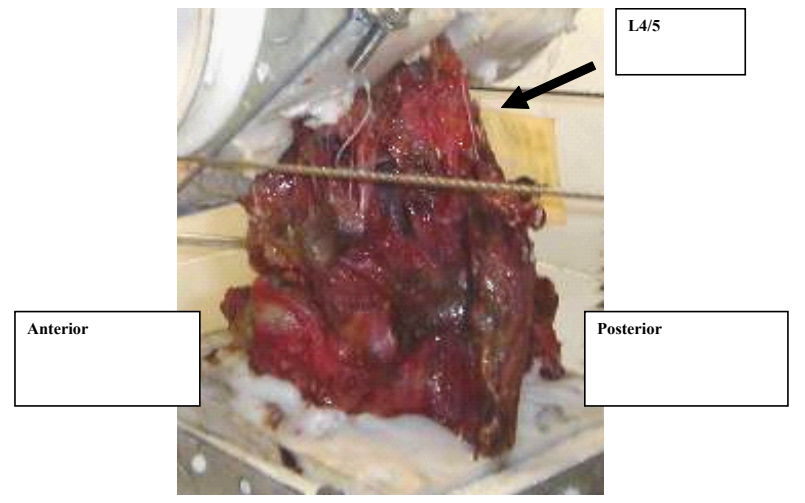

Figure 5: Complete failure of posterior ligamentous complex at $L 4 / 5$ with failure testing. Note no failure at L5/S1.

\section{Results}

The seven specimens tested were harvested from cadavers with an average age of 74.8 years, (range 56 to 94), and the specimens had an average BMD of -3.4 , (range -2.5 to -4.9 ).

The data for the cyclic testing is found in Table 1, while the data for destructive testing is shown in Table 2. During the cyclic tests the mean displacement of L5 relative to $\mathrm{S} 1$ in the AxiaLIF group was 0.26 $\pm 0.15 \mathrm{~mm}(\mathrm{n}=3)$, and $0.09 \pm 0.09 \mathrm{~mm}$ in the pedicle screw group $(\mathrm{n}=$ 4). During the failure tests the mean displacement of L5 relative to S1 was $3.74 \pm 3.34 \mathrm{~mm}$ in the AxiaLIF group $(\mathrm{n}=3)$ and $4.57 \pm 4.14$ in the pedicle screw group $(n=4)$. A univariate analysis of variance was used to compare the mean displacement of L5 relative to $\mathrm{S} 1$ between the two groups in both cyclic testing, and in testing to failure. No significant differences were observed between the relative displacement of L5 on $\mathrm{S} 1$ in either cyclic testing $(\mathrm{p}=0.15)$ or testing to failure $(\mathrm{p}=0.61)$.

No overt failure was noted in any specimen through the L5/S1 segment. However, surprisingly in 3 specimens there was complete failure of the posterior interspinous ligament, facet capsule and posterior annulus at the L4/5 disc space above the construct. This occurred in two of the axial rod constructs and one of the pedicle screw constructs (Figure 5).

\section{Discussion}

Osteoporosis and degeneration may co-exist in the same patient and may necessitate stabilization. A difficulty in treating these patients is the lack of sufficient bone in which to instrument and apply corrective forces to the spine. Perhaps this problem is most pronounced at the L5S1 junction as it is the site of the highest shear forces given its anatomic obliquity and the primary site for lumbar spinal lordosis. Rigid fixation at an osteoporotic L5-S1 level is particularly difficult because it is at the spine terminus where no further spinal distal fixation points exist for additional reinforcement. To successfully achieve L5-S1 fusion, many spinal surgeons instrument the pelvis and distal sacrum for additional reinforcement and include it in the fusion, thus taking away all motion in the lower spine $[3,4]$. While this approach does increase the stability, it also is associated with extensive blood loss, increased morbidity, longer recovery periods, and most significantly, catastrophic construct failure [5]. This reconstruction strategy is also problematic because caudal screws (particularly at L5-S1) are subjected to greater loads than rostral screws in multi segmental constructs. When screws at the L5-S1 junction fail, there is failure of the entire construct as the main anchor (at the L5-S1 level) of the stabilizing instrumentation is lost and sacral fracture occurs. When the instrumentation fails at L5-S1, very few options exist for salvage or deformity correction. The patient is then left with chronic, disabling pain and potential neurologic injury associated with a deformed and unstable spine.

With the recent advent of less invasive surgery, conventional thoughts and techniques regarding spinal instrumentation in osteoporotic bone, for instance, more points of fixation, are now being questioned. The use of strategic, biomechanically favorable implants may, in fact, be clinically superior in the stabilization of an osteoporotic L5-S1 junction.

The L5-S1 trans-sacral axial rod represents a less invasive approach to spinal fixation [6,7]. It provides effective fixation of the lumbosacral junction without disruption of the stabilizing posterior structures of the spine and the annulus of the intervertebral segment. This device is designed to be implanted through a percutaneous paracoccygeal transsacral approach. In this fashion there is no disruption of the muscular attachments of the spine and thus prevents the pain and morbidity associated with traditional lumbosacral fixation with pedicle screws where there is a large dissection and extensive muscle detachment [8]. The trans-sacral axial rod is a load sharing device with one half embedded in the L5 vertebrae and the other half into the sacrum. This form of rigid fixation and its perpendicular position theoretically offers excellent axial strength while resisting motion due to its position outside the instantaneous axis of rotation. However, there are no biomechanical studies comparing the trans-sacral axial rod to the standard pedicle screw construct at L5-S1 in osteoporotic bone.

Several non-osteoporotic models have been tested. Bovine cadaveric studies have shown the trans-sacral rod to be superior to stand alone anterior devices in lateral bending and axial rotation [9]. One study looked at the strength of the trans-sacral rod in human cadaveric non-osteoporotic spines in non destructive testing. They found that the trans-sacral rod significantly decreased motion in all three dimensions (axial rotation, lateral bending and flexion/extension). The addition of posterior instrumentation increased the stiffness of the construct in all three motions, but with less of an effect with flexion/ extension [10]. Lengthening the trans-sacral rod from L4-S1, posterior supplementation had a significant effect at L5/S1 in flexion/extension and at $\mathrm{L} 4 / 5$ in lateral bending [11].

In our biomechanical study, there was no statistical difference between the axial rod construct and the pedicle screw construct for motion at L5/S1, similar to those found previously [8]. This was found with cyclical testing as well as with destructive testing. Unexpectedly, we found that in three specimens we generated failure with significant displacement $(>17 \mathrm{~mm})$ at the $\mathrm{L} 4 / 5$ disc interspace. This occurred due to complete failure of the posterior ligamentous complex at the L4/5 level. Osteoporosis did not seem to play a role as failure occurred through the soft tissue rather than failure through the bone. This data suggests that the loads applied in this biomechanical model were superphysiologic. It also demonstrates that despite these super-physiologic loads we did not demonstrate failure of the trans-sacral axial rod at the L5/S1 motion segment.

There are several limitations to our biomechanical experiment. First the biomechanics represented may not represent what occurs clinically. Although our apparatus allowed for pure flexion and extension to occur, this is likely not the only forces that are resisted by the implants clinically. However, the pure flexion moment allowed the specimen to translate and allow the center of rotation to change with additional flexion and extension. The two models were also biomechanically disadvantaged models, and clinically surgeons may 
choose to place anterior support in the pedicle screw construct and posterior instrumentation in the trans-sacral rod construct. The final limitation is the small number of specimens used in each group, and although there was no statistical differences, with larger numbers subtle differences may be found.

\section{Conclusion}

The trans-sacral axial rod construct was found to be comparable to pedicle screws in terms of absolute stability at the L5/S1 disc space with a pure flexion moment. In addition, with destructive testing no overt failure could be demonstrated at the instrumented levels, even while applying super-physiologic loads. Despite the implications of these findings, ultimate strength of the construct may not be the most important factor in achieving either a successful clinical outcome, or a successful fusion. The limitations of this study are that its conclusions only apply to pure flexion moments and no assumptions should be made for rotation or lateral bending in osteoporotic bone. Clinical studies are needed to asses for fusion and outcome in a randomized fashion.

\section{References}

1. Glassman SD, Alegre GM (2003) Adult spinal deformity in the osteoporotic spine: options and pitfalls. Instr Course Lect 52: 579-588.

2. DeWald CJ, Stanley T (2006) Instrumentation-related complications of multilevel fusions for adult spinal deformity patients over age 65: surgical considerations and treatment options in patients with poor bone quality. Spine 31: S144-S151.
3. Santos ER, Rosner MK, Perra JH, Polly DW Jr (2007) Spinopelvic fixation in deformity: a review. Neurosurg Clin N Am 18: 373-384.

4. Tsuchiya K, Bridwell KH, Kuklo TR, Lenke LG, Baldus C (2006) Minimum 5-year analysis of L5-S1 fusion using sacropelvic fixation (bilateral S1 and iliac screws) for spinal deformity. Spine 31: 303-308.

5. Jutte PC, Castelein RM (2002) Complications of pedicle screws in lumbar and lumbosacral fusions in 105 consecutive primary operations. Eur Spine $\mathrm{J} 11$ : 594-598.

6. Marotta N, Cosar M, Pimenta L, Khoo LT (2006) A novel minimally invasive presacral approach and instrumentation technique for anterior L5-S1 intervertebral discectomy and fusion: technical description and case presentations. Neurosurg Focus 20: E9.

7. Aryan HE, Newman CB, Gold JJ, Acosta FL Jr, Coover C, et al. (2008) Percutaneous axial lumbar inter-body fusion (AxiaL IF) of the L5-S1 segment: initial clinical and radiographic experience. Minim Invasive Neurosurg 51: 225230.

8. Yuan PS, Day TF, Albert TJ, Morrison WB, Pimenta L, et al. (2006) Anatomy of the percutaneous presacral space for a novel fusion technique. J Spinal Disord Tech 19: 237-241.

9. Ledet EH, Tymeson MP, Salerno S, Carl AL, Cragg A (2005) Biomechanical evaluation of a novel lumbosacral axial fixation device. J Biomech Eng 127 929-933.

10. Akesen B, Wu C, Mehbod AA, Transfeldt EE (2008) Biomechanical evaluation of paracoccygeal transsacral fixation. J Spinal Disord Tech 21: 39-44.

11. Erkan S, Wu C, Mehbod AA Hsu B, Pahl DW, et al. (2009) Biomechanical evaluation of a new AxiaLIF technique for two-level lumbar fusion. Eur Spine J 18: 807-814 